\title{
Cobertura y estado de los arrecifes de coral e ictiofauna asociada en Playa Cacique e Isla Mamey, Portobelo, Colón
}

\section{Coverage and status of coral reefs and ichthyofauna associated in Cacique beach and Mamey island, Portobelo, Colón}

\author{
Ramón Guerra ${ }^{1 *}$, Aramis Aparicio ${ }^{1}$, Benjamín Espinosa ${ }^{1}$, José Julio Casas ${ }^{1}$ \\ ${ }^{I}$ Facultad de Ciencias del Mar_Universidad Marítima Internacional de Panamá
}

\begin{abstract}
Resumen Los arrecifes de coral son comunidades de protección de la zona costera y alimentación de muchas especies. Es importante evaluar el estado de estos, para así poder proteger y mantener la salud de los ecosistemas tan importantes. Este trabajo se realizó en Playa Cacique e Isla Mamey en Colón (Caribe Panameño), en dos localidades con actividades humanas diferentes. Se realizaron dos salidas al campo con cuatro días de campo con 12 horas en total. En cada estación se establecieron transectos al azar de $10 \mathrm{~m}$ de largo perpendiculares a la costa. Los resultados arrojaron buenos porcentajes de coral vivo para ambas áreas de estudio, hubo una diversidad de 14 especies de corales, siendo la familia Faviidae la más diversa. En comparación realizada se notó un $41 \%$ de coral vivo en la estación de Mamey, pero Cacique posee más vegetación. Además, Cacique posee 13 de las 14 especies de corales. Los arrecifes, en su mayoría estaban representados por corales fuego en los transectos tres y cuadro de Cacique y uno y dos de Mamey, representadas por aguas someras. Además, el transecto cuatro de Mamey y uno de Cacique presentaron mayor diversidad de especies de corales. En cuanto a la abundancia y diversidad de ictiofauna asociada, no presentó una diferencia significativa entre las dos áreas de estudio, presentando una diversidad de 21 especies y 11 familias, siendo Halichoeres pictus la especie más común, y Labridae la familia más diversa. Realizamos el índice de Simpson para la diversidad de peces en las dos áreas de estudio, dando como resultado: Playa Cacique (0.745) e Isla Mamey (0.812), y la varianza fue de 1.01, teniendo Mamey la mayor diversidad de especies de peces. Como conclusiones, las especies de Coral de fuego se presentan y colonizan las aguas someras de Playa Cacique e Isla Mamey, y esta última zona presentó una alta diversidad de especies de coral, pero los corales de Cacique se mantienen en mejor estado, al tener un ecosistema de manglares que mitiga el sedimento hacia los corales. La especie Halichoeres pictus se presentó en la mayoría de los transectos, debido a su adaptación a aguas con fuertes oleajes. Se debe hacer un esfuerzo para mitigar las actividades antropogénicas de estas dos localidades y aumentar el estudio de los corales y peces asociados en el area.
\end{abstract}

Palabras clave Arrecifes de coral, peces de arrecife, monitoreo de arrecifes.

\begin{abstract}
Coral reefs are communities of protection of the coastal zone and feeding of many species. It is important to evaluate the state of coral reefs, in order to protect and maintain the health of these important ecosystems. This work was carried out in Playa Cacique and Isla Mamey in Colón (Caribbean cost of Panama), in two locations with different human activities. Two field trips were made with four field days with a total of 12 hours. At each station, 10-meter-long random transects were established perpendicular to the coast. The results yielded good percentages of live coral for both study areas, there was a diversity of 14 coral species and Faviidae being the most diverse family, and a comparison made $41 \%$ of live coral was noticed in the Mamey station, but Cacique has more vegetation, and Cacique has 13 of the 14 coral species. The reefs were mostly represented by fire corals in transects three and four in Cacique and transects one and two in Mamey, represented by shallow water. In addition, transect four in Mamey and one in Cacique presented a greater diversity of coral species. Regarding the abundance and diversity of associated ichthyofauna, there was no significant difference between the two study areas, presenting a diversity of 21 species and 11 families, Halichoeres pictus being the most common species, and Labridae the most diverse family. We performed the Simpson Index for fish diversity in the two study areas, resulting in: Cacique Beach (0.745) and Mamey Island (0.812), and the variance was 1.01, with Mamey having the greatest diversity of fish species. As conclusions, the Fire Coral species are presented and colonize the shallow waters of Cacique Beach and Mamey Island, and this last area presented a high diversity of coral species. However, Cacique corals are maintained in a better state, having a Mangrove ecosystem that mitigates sediment towards corals. The Halichoeres pictus species was present in most of the transects, due to its adaptation to waters with strong waves. An effort must be made to mitigate the anthropogenic activities of these two localities and to increase research on corals and associated fish in the area.
\end{abstract}

Keywords Coral reefs, reef fish, reef monitoring. 


\section{Introducción}

Los arrecifes coralinos son comunidades complejas construidas por el crecimiento constante de corales, algas coralinas incrustantes, y otros organismos calcáreos, habitados por cerca del $25 \%$ de todas las especies marinas [1]. Estos ecosistemas son hábitats importantes en la zona costera tropical, donde forman barreras paralelas que protegen la costa [2]. Estas comunidades originan estructuras sólidas resistentes al oleaje y tormentas [3-6]. Además, son áreas de pesquerías de subsistencia en el Mar Caribe y Océano Pacífico, con un alto valor económico, cultural y recreacional [7-10].

Los corales pueden ser de dos tipos, ahermatípicos (blandos) y hermatípicos (duros), estos últimos también llamados escleractínidos son los mayores formadores de arrecifes, además, poseen algas simbióticas llamadas zooxantelas que al desaparecer ocasionan el blanqueamiento del coral [11]. La expulsión de las zooxantelas ocurre cuando el coral se encuentra bajo algún tipo de presión ambiental [12], por ejemplo, presencia de agua dulce en un arrecife [13], temperatura alta [14], o cuando están expuestos a ataques de patógenos que blanquean los corales [15-20], disminuyendo así la diversidad y abundancia a nivel global [21]. Los arrecifes más cercanos a la costa son los más vulnerables [22] ante perturbaciones por: tránsito marítimo, contaminación, sobrepesca y turismo [23]. También el incremento de las tasas de sedimento y nutrientes provenientes de la agricultura [2425] han sido las causas principales de esta degradación mundial [2].

Según diferentes autores [26, 27], la riqueza de organismos del arrecife de coral es comparable con la del bosque tropical lluvioso; por lo que se consideran entre los ecosistemas más productivos y diversos de la tierra [9]. Los peces de los arrecifes de coral son las comunidades más diversas de vertebrados en la tierra [28] y su distribución espacio-temporal está influenciada por una combinación de complejos biológicos y factores físicos. Estos factores incluyen el suministro de larvas [29], la competencia [30-31], la exposición a las olas [32], la profundidad [33] y la complejidad del hábitat [34].

Los peces de arrecife, como depredadores o herbívoros, juegan un papel importante en la dinámica de las comunidades arrecifales, a través de sus interacciones con los corales, algas, otros herbívoros y otros depredadores [35]. La ruptura en el equilibrio de las poblaciones de herbívoros de arrecife puede disminuir la cobertura de coral y aumentar la abundancia de algas [36], [37]. Debido a esta importancia, los peces son a menudo un foco de monitoreo y programas de gestión para evaluar la condición de las comunidades de arrecifes [38]. Históricamente los peces de arrecife de coral han sido difíciles de estudiar, en parte, porque las especies a menudo tienen diferentes requerimientos de hábitat [39], patrones de vida [40] y regímenes alimenticios [41].

Los primeros estudios de comunidades coralinas en ambas costas de Panamá se realizaron a comienzos de la década de los setenta [42-44]. Se ha concluido que las interacciones entre las dos vertientes del país provocan unos efectos adversos para las comunidades arrecifales cercanas a la cuenca del Canal de Panamá, impulsado más investigaciones para monitorear el estado, ya que la información de la época era muy escasa [45]. Estudios más recientes han revelado nuevas especies de coral para ambas costas de Panamá, algunas de estas representadas en una sola población de pocos individuos [46-49] y [9]. En el Caribe panameño se encuentran 64 especies de corales escleractínidos e hidrocorales [50].

Los arrecifes de coral proveen bienes y servicios ecosistémicos para el turismo, la pesca y la protección del litoral. Cerca del $60 \%$ de las zonas de arrecife coralino se sitúan en países en desarrollo, en los que cerca de seis millones de personas dependen enormemente de los servicios que prestan estos ecosistemas. Se ha estimado el valor económico global de los arrecifes de coral en hasta 375 mil millones de dólares por año [51].

Panamá cuenta con diferentes normativas para la conservación y uso de estos ecosistemas como es la ley $\mathrm{N}^{\circ} 14$ del 28 de octubre de 1977, que aprobó la convención sobre el comercio internacional y especies amenazadas de fauna y flora silvestre (CITES). Dentro de este convenio, en el apéndice II se encuentran el orden de los escleractínidos y la familia milleporidae. Esta también se apoya en la resolución J.D. 033 de 28 de septiembre de 1993 (G.O. 22430 de 10/12/93), que prohíbe la extracción y exportación de todas las especies de corales a nivel nacional con fines comerciales, ya sea vivas o muertas, con excepción de las obtenidas con fines científicos previo permiso de la autoridad [52]. Otra ley de importancia es la ley $\mathrm{N}^{\circ} 32$ del 9 de febrero de 1996, que estipula en el artículo $\mathrm{N}^{\circ} 1$ que los arrecifes de corales y los corales se vuelven inadjudicables, a excepción de los corales muertos. El uso de estos ecosistemas se regula mediante la Ley 2 de 7 de enero de 2006, que regula las concesiones para la inversión turística y la enajenación de territorio insular para fines de su aprovechamiento turístico y dicta otras disposiciones. Esta ley establece dentro de su ámbito disposiciones protectoras de estos ecosistemas; en su artículo 32, queda prohibida la construcción sobre las formaciones de coral, así como cualquier actividad que produzca su muerte, blanqueo o la destrucción de los ecosistemas de los que son parte. La gestión de los recursos marino-costeros será regulada a través de disposiciones que para este fin realice la Autoridad Marítima 
de Panamá en coordinación con la Autoridad Nacional del Ambiente.

También los peces que habitan estos ecosistemas están amparados por el decreto Ejecutivo 29 de 24 de junio de 1994 (G.O. 22576 de 11/07/94), que prohíbe la captura y exportación de peces asociados con áreas coralinas en la Zona Económica Exclusiva de Pesca de Panamá, en el Océano Pacífico y en el Océano Atlántico [52].

El objetivo general de esta investigación fue determinar el estado de los arrecifes de coral y su ictiofauna asociada, en Playa Cacique e Isla Mamey, ya que estos son el principal ingreso económico de estas áreas.

\section{Metodología}

\section{1 Área de estudio}

El estudio fue realizado en Cacique $\left(9^{\circ} 37^{\prime} 0.12^{\prime \prime} \mathrm{N}, 79^{\circ} 37^{\prime}\right.$ $0.12^{\prime \prime} \mathrm{W}$ ) un corregimiento con una superficie del territorio de $11.4 \mathrm{~km}^{2}$ [53]; que limita al Norte y al Oeste con el Mar Caribe, al Sur con el corregimiento de Portobelo (cabecera) y al Este con el corregimiento de Garrote e Isla Mamey $\left(9^{\circ} 37^{\prime} 00.0^{\prime \prime} \mathrm{N}\right.$ $\left.79^{\circ} 36^{\prime} 00.0^{\prime \prime} \mathrm{W}\right)$ que forma parte del corregimiento de Garrote, ambas pertenecientes al distrito de Portobelo.

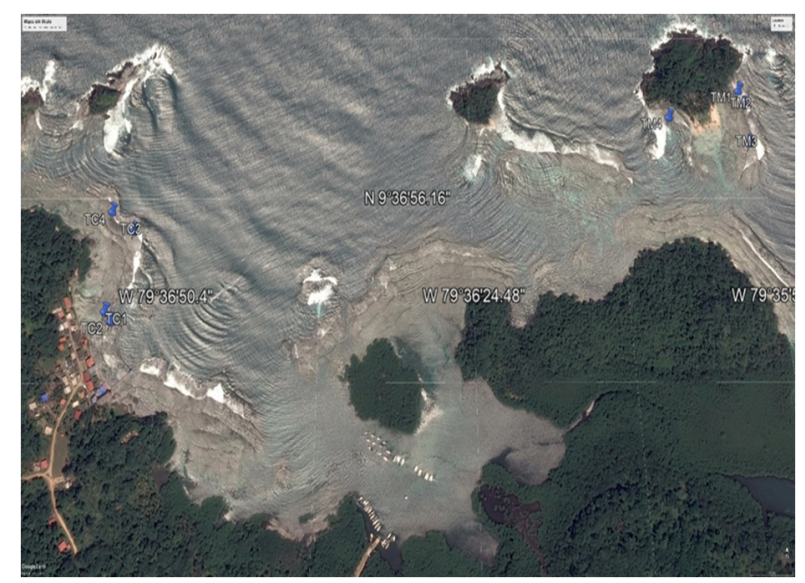

Figura 1. Mapa del área de estudio con los transectos realizados marcados en azul.

Ambas zonas se encuentran dentro de la jurisdicción del Parque Nacional Portobelo creado mediante el Decreto 91 del 22 de diciembre de 1976 . La zona marina del parque abarca 8 mil 37 hectáreas $(22.37 \%)$ de sus 35,929 hectáreas de extensión; posee hermosos arrecifes coralinos, los cuales se desarrollan en forma de parches a lo largo de 250 kilómetros de las costas e islas [54].

Cacique, posee una población de 273 personas con una densidad de 23.9 habitantes por $\mathrm{km}^{2}$ [55]. La principal actividad de la zona es la agricultura de subsistencia, principalmente granos, tubérculos y frutos. Adicionalmente, realizan actividades extractivas, de interés pesquero, madera para leña y construcción, plantas y cueros para la producción de artesanías e instrumentos musicales, así como también realizan pesca artesanal [56]. Por su parte, Mamey es una Isla deshabitada, donde se desarrollan actividades recreativas [57].

En estas zonas el clima es tropical, las temperaturas tienen promedio de $32^{\circ} \mathrm{C}$ de día y $23^{\circ} \mathrm{C}$ de noche [58]. Las precipitaciones son mucho más abundantes en temporada seca que en lluviosa, con una media anual de pluviosidad de $3553 \mathrm{~mm}$ [59]. Según las zonas de vida de Holdridge [60], esta área presenta un bioma de tipo bosque húmedo. En relación con las características geológicas, Portobelo y sus alrededores se caracterizan por rocas de origen volcánico muy cizalladas y fracturadas, las cuales se clasifican como tobas y lavas andesíticas y basálticas. Presenta fuerte acción tectónica, y por la actividad sísmica reciente, se cree que tal vez está en un área tectónica regional y posiblemente es una zona de subducción.

La topografía es irregular con predominancia de lomas y cerros con pendientes mayores de $45^{\circ}$ [57].

\subsection{Materiales y métodos}

Se realizaron dos salidas al campo, utilizando la metodología de AGRRA adaptada a las condiciones que se presentaban en el área de estudio. Definimos las dos estaciones: Playa Cacique (TC) e Isla Mamey (TM). Cada estación fue dividida al azar en cuatro transectos de siete metros de largo por uno de ancho, perpendiculares a la costa, los cuales se midieron con una cinta métrica. Se tomaron las coordenadas de cada transecto con un GPS Garmin, luego se introdujeron en la aplicación Garmin para luego trasladarlas a Google Earth para georreferenciar los transectos.

\subsubsection{Cobertura de los arrecifes}

Ya establecidos los transectos, se utilizó una cuadrícula de $1 \mathrm{~m}^{2}$ para calcular la cobertura, en porcentajes, de los arrecifes. Esto se hizo gracias a que las cuadrículas están divididas por dentro con unos hilos de nylon. La cobertura se calculó usando los cuadros dentro de la cuadrícula, viendo que la cantidad de cuadros llenos por esta; se clasificó la cobertura en: CV (coral vivo), CM (coral muerto), A (algas), AI (alga incrustante), HM (hierba marina) y D (roca viva). Se registraron en una tabla los porcentajes de cobertura de cada cuadrícula por transecto (siete cuadrículas por transecto), para luego promediarlas y así calcular la cobertura por transecto. Para calcular la cobertura de cada estación, se promediaron los transectos. Como hicimos dos salidas, se promediaron los resultados de cada una para tener una media en la cobertura.

\subsubsection{Estado de los arrecifes}

Se observó el estado en que se encontraban, clasificándolos en: $\mathrm{S}$ (sano) cuando el coral no presentaba ninguna afectación, $\mathrm{E}$ (enfermo) cuando presentaba un sobrecrecimiento de algas y/o una enfermedad como puede ser las bandas (negra, blanca,

\section{RIC}


etc.), B (blanqueamiento) cuando presentaba un blanqueamiento parcial o total y $\mathrm{R}$ (roto) cuando presentaba un daño en su morfología. Para determinar el estado de cada transecto se promedió el porcentaje.

\subsubsection{Abundancia y diversidad de corales}

Se identificaron las diferentes especies de coral presentes en los transectos usando la técnica de foto-identificación, con una cámara Canon Powershot D30, y con la ayuda de sitio web Coralpedia [61]. Se determinó el transecto más diverso (el que más especies presentaba por estación), como también la familia más abundante (la que se encontraba en mayor cantidad).

\subsubsection{Abundancia y diversidad de peces}

Paralelo al monitoreo de los Corales, realizamos el monitoreo de la ictiofauna asociada al arrecife. Para la identificación de las especies se tomaron fotografías in situ, que fueron revisadas en los laboratorios de UMIP, con el App Peces: Gran Caribe [62]. Para calcular la diversidad de especies se utilizó el índice de Simpson que indica el nivel de biodiversidad en cada estación, luego se calculó la varianza para comparar las estaciones. Es importante mencionar que en ninguna de las giras se extrajeron organismos de ninguna especie.

\section{Resultados}

Cada estación constó de cuatro transectos escogidos al azar.

El primer monitoreo se realizó con condiciones poco óptimas, ya que la marea estaba más baja de lo usual, dejando al descubierto muchos corales. También se presentó una turbiedad inusual en el área. Para el segundo monitoreo se presentó un oleaje fuerte lo que impidió poder realizar el transecto 2 en Isla Mamey. Se muestréo un total de $56 \mathrm{~m}^{2}$ de superficie entre los ocho transectos, en promedio se trabajaron tres horas por salida, con un total de 12 horas efectivas de muestreo para todo el proyecto.

\subsection{Cobertura de los arrecifes}

Los porcentajes de cobertura de los arrecifes en Playa Cacique estuvo distribuida en su mayoría en: coral vivo (29\%), roca viva $(28 \%)$ y algas $(27 \%)$. El transecto con mayor porcentaje de cobertura de coral vivo en Cacique fue el segundo, en Mamey el cuarto. Playa Cacique e Isla Mamey poseen praderas marinas, las cuales contribuyen a mitigar la sedimentación en los arrecifes coralinos. Solo se encuentran manglares en Playa Cacique. Los transectos TC1, TC2 y TM4 fueron los únicos con profundidades mayores a cinco pies, los demás solo llegaban a un máximo de dos pies. A diferencia de Playa Cacique, en Isla Mamey no se encontraron hierbas marinas en los transectos realizados.
Tabla 1. Cobertura de arrecife de coral en los transectos de Playa Cacique e Isla Mamey

\begin{tabular}{|c|c|c|c|c|c|c|}
\hline Transecto & CV & CM & A & AI & HM & D \\
\hline TC1 & 18 & 0 & 34 & 5 & 17 & 26 \\
\hline TC2 & 46 & 0 & 20 & 11 & 0 & 23 \\
\hline TC3 & 27 & 2 & 13 & 16 & 1 & 41 \\
\hline TC4 & 25 & 0 & 43 & 9 & 1 & 23 \\
\hline TM1 & 44 & 0 & 14 & 11 & 0 & 31 \\
\hline TM2 & 34 & 0 & 29 & 9 & 0 & 28 \\
\hline TM3 & 37 & 0 & 6 & 14 & 0 & 43 \\
\hline TM4 & 48 & 0 & 10 & 10 & 0 & 32 \\
\hline
\end{tabular}

Las siglas representan: TC (transecto de Cacique), TM (transecto de Mamey), $\mathrm{CV}$ (coral vivo), CM (coral muerto), A (algas), AI (alga incrustante), HM (hierba marina) y D (roca viva).

En esta comparación de las dos estaciones Playa Cacique e Isla Mamey (ver figura 2); se puede notar que en Mamey predominó en la cobertura de coral vivo con un promedio de $41 \%$, pero cacique posee una mayor cobertura de vegetación (algas y hierbas marinas) que Isla Mamey, a su vez, podemos observar que las algas incrustantes de ambas estaciones están en un porcentaje similar.

$$
m \mathrm{CV}=\mathrm{CM} \equiv \mathrm{A}=\mathrm{Al} \equiv \mathrm{HM} \equiv \mathrm{D}
$$

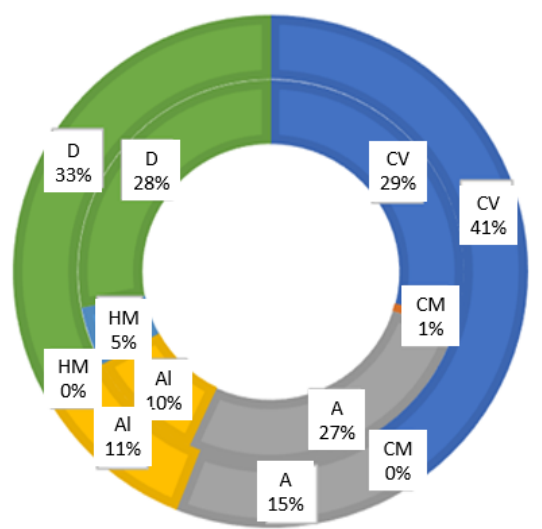

Figura 2. Gráfica comparativa de la cobertura de los arrecifes presente en las estaciones. El círculo interior representa a Playa Cacique y el externo a Isla Mamey.

\subsection{Estado de los arrecifes}

A pesar de que Cacique presenta un mayor porcentaje de cobertura de coral vivo, estos presentan un alto grado de afectación. Los resultados indican que los últimos transectos, obtuvieron un mejor porcentaje en su estado. Isla Mamey presentó una cantidad reconocida de blanqueamiento de coral, en cambio, en los corales rotos hubo un mínimo porcentaje en TM3, con 14\%. En Isla Mamey, los corales presentaban mayor sobre crecimiento de algas. 
Tabla 2. Estado de los arrecifes de coral en los transectos de Playa Cacique e Isla Mamey

\begin{tabular}{|c|c|c|c|c|}
\hline Transecto & S & E & \multicolumn{1}{|c|}{ B } & R \\
\hline TC1 & 25 & 50 & 0 & 25 \\
\hline TC2 & 29 & 43 & 14 & 14 \\
\hline TC3 & 72 & 14 & 0 & 14 \\
\hline TC4 & 72 & 28 & 0 & 0 \\
\hline TM1 & 72 & 28 & 0 & 0 \\
\hline TM2 & 29 & 57 & 14 & 0 \\
\hline TM3 & 0 & 72 & 14 & 14 \\
\hline TM4 & 43 & 57 & 0 & 0 \\
\hline
\end{tabular}

Las siglas representan: TC (transecto de Cacique), TM (transecto de Mamey), S (sano), E (enfermo), B (blanqueamiento) y R (roto).

Los resultados indican que Mamey posee un peor estado de conservación comparado con Cacique, donde el porcentaje de coral enfermo supera el 50\%. En Cacique el 50\% de los corales están sanos. Sin embargo, aproximadamente el 13\% están rotos. Se observó un bajo porcentaje de blanqueamiento en las estaciones, solo se reporta un $10 \%$ de corales blanqueados para ambos sitios ver figura 3 .

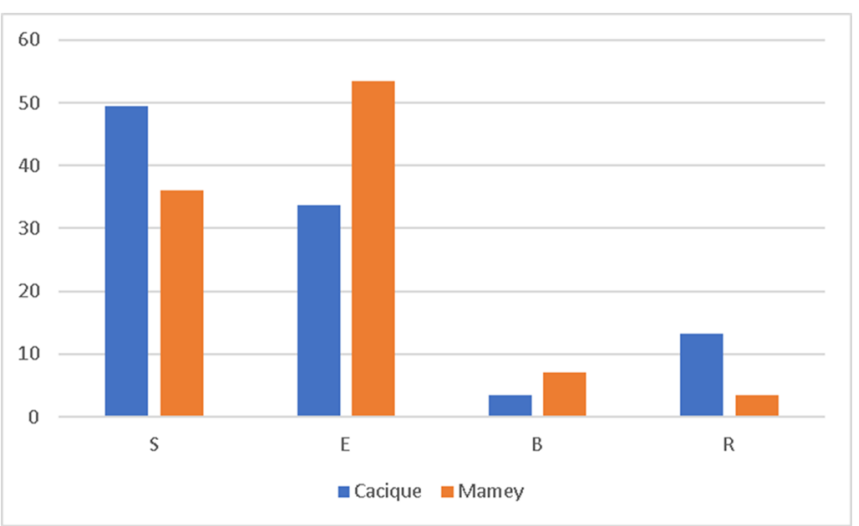

Figura 3. Gráfica comparativa del estado de las estaciones.

\subsection{Abundancia y diversidad de corales}

Se encontraron un total de 12 corales escleractínidos y dos hidrocorales, siendo estos últimos los más abundantes. El transecto que presentó mayor diversidad de especies fue el TC1 con siete de las 14 especies encontradas, seguido del TM4 con seis especies. Solo se encontró un transecto monoespecífico, el TM1. El coral cuerno de alce (Acropora palmata) solo se encontró en Isla Mamey. Cacique posee 13 de las 14 especies de corales, en cambio, Mamey solo cuenta con nueve.
Tabla 3. Diversidad y abundancia de arrecifes de coral en Playa Cacique e Isla Mamey

\begin{tabular}{|c|c|c|c|}
\hline Clase & Orden & Familia & Especie \\
\hline \multirow{12}{*}{ Anthozooa } & \multirow{12}{*}{ Scleractinia } & Acroporidae & Acropora palmata \\
\hline & & \multirow{2}{*}{ Agariciidae } & Agaricia agaricites \\
\hline & & & Agaricia tenuifolia \\
\hline & & \multirow{5}{*}{ Faviidae } & Colpophyllia natans \\
\hline & & & Diploria clivosa \\
\hline & & & Diploria strigosa \\
\hline & & & Favia fragum \\
\hline & & & Orbicella faveolata \\
\hline & & Meandrinidae & Dichocoenia stokesii \\
\hline & & Poritidae & Porites porites \\
\hline & & \multirow{2}{*}{ Siderastreidae } & Siderastrea radians \\
\hline & & & Siderastrea sidérea \\
\hline \multirow{2}{*}{ Hydrozoa } & \multirow{2}{*}{ Anthoathecata } & \multirow{2}{*}{ Milleporidae } & Millepora complanta \\
\hline & & & Millepora striata \\
\hline
\end{tabular}

\subsection{Abundancia y diversidad de peces}

En las estaciones de Playa Cacique e Isla Mamey se encontraron un total de 21 especies de peces pertenecientes a cuatro órdenes (Anguiliformes, Perciformes, Scorpaeniformes, y Tetraodontiforme) y 11 familias. Los resultados revelan que el orden más abundante y diverso fue el perciforme. A su vez, la familia más diversa fue Labridae con seis especies, seguida de la Familia Acanthuridae y Scaridae con tres especies cada una. La especie Halichoeres pictus fue la más común presente en tres de los cuatro transectos en Cacique y tres de los cuatro transectos en Mamey; además, también es reconocida por ser la más abundante con una cantidad de 30 individuos en promedio, la segunda especie más común fue Stegastes diencaeus que se encontró en tres de los cuatro transectos en Cacique y uno en los cuatro transectos en Mamey (ver tabla 4).

Tabla 4. Abundancia y diversidad de peces en Playa Cacique e Isla Mamey

\begin{tabular}{|c|c|c|}
\hline Orden & Familia & Especie \\
\hline Anguiliforme & Muraenidae & Echidna catenata \\
\hline \multirow{7}{*}{ Perciforme } & \multirow{4}{*}{ Acanthuridae } & Acantharus helioides \\
\cline { 3 - 3 } & & Acanthurus chirurgus \\
\cline { 3 - 3 } & Chaenopsidae & Acanthurus coeruleus \\
\cline { 3 - 3 } & Haemulidae & Haemulon carbonarium \\
\cline { 3 - 3 } & & Halichoeres bivittatus \\
\cline { 3 - 3 } & \multirow{4}{*}{ Labridae } & Halichoeres maculipinna \\
\cline { 3 - 3 } & & Halichoeres pictus \\
\cline { 3 - 3 } & & Halichoeres radiatus \\
\hline
\end{tabular}




\begin{tabular}{|c|c|c|}
\hline \multirow{7}{*}{} & & Thalassoma purpureum \\
\cline { 3 - 3 } & \multirow{4}{*}{ Pomacentridae } & Thalassoma bifasciatum \\
\cline { 3 - 3 } & & Stegastes leucostictus \\
\cline { 3 - 3 } & Serranidae & Stegastes diencaeus \\
\cline { 3 - 3 } & & Epinephelus itajara \\
\hline Scorpaeniforme & Scorpaenidae & Scarus iseri \\
\cline { 3 - 3 } & & Sparisoma rubripinne \\
\hline \multirow{2}{*}{ Tetraodontiformes } & Diodontidae & Diodon \\
\cline { 3 - 3 } & Tetraodontidaerum & Canthigaster rostrata \\
\hline
\end{tabular}

Cacique presentó un total de 130 individuos y 13 especies, mientras que para Mamey se reportan 140 individuos y 15 especies. El índice de diversidad Simpson para las estaciones dieron los siguientes resultados: Playa Cacique $(0.745)$ e Isla Mamey (0.812). La varianza fue de 1.01. Teniendo así, una mayor diversidad de peces en Isla Mamey en los días de muestreo.

Mamey presenta una mayor abundancia y diversidad de especies de peces que Cacique, donde ocho especies solo se observaron en esta zona, incluyendo la morena. Por otro lado, en Cacique se reportan cinco especies observadas solo para la zona durante los muestreos, entre los que se menciona dos especies de pez loro (ver figura 4).

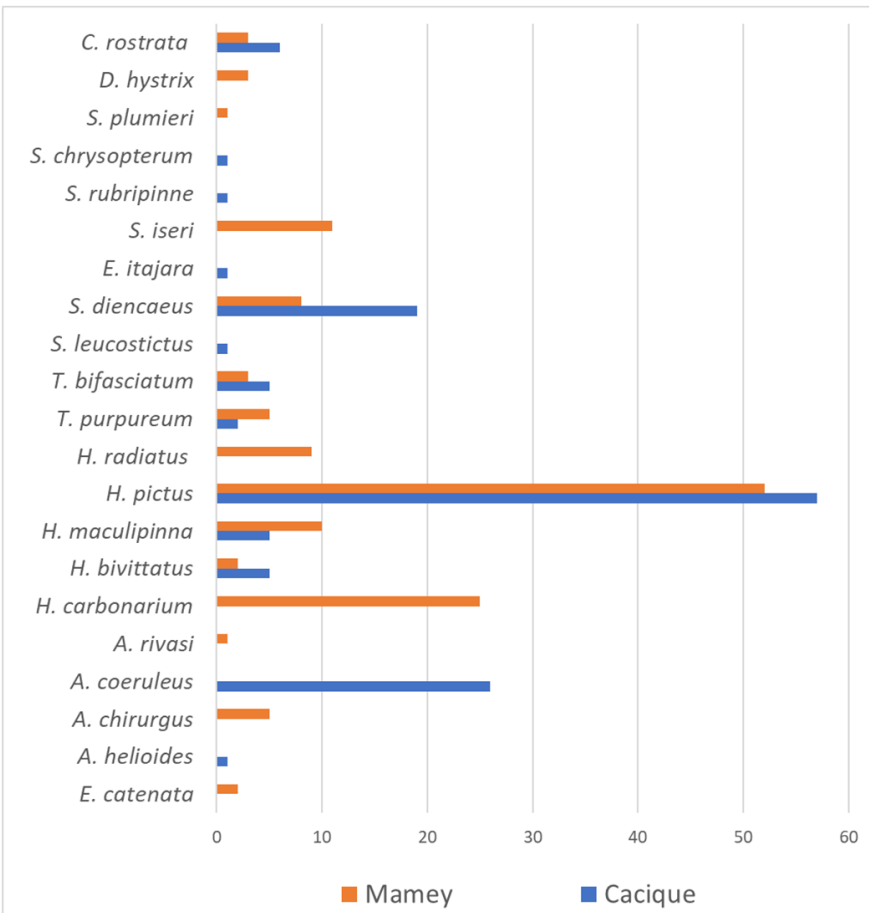

Figura 4. Abundancia y diversidad de peces presentes en las estaciones de muestreo.

Vol. 5 - N. ${ }^{\circ}$ Especial I $\cdot$ Marzo $\cdot 2019$

\section{Discusión}

El porcentaje de cobertura de coral vivo de Cacique fue de $29 \%$ en comparación con el $41 \%$ en Mamey, es decir un $12 \%$ de cobertura de coral, se sugiere que debido a que Mamey al ser una Isla las presiones antropogénicas están menos presentes que en tierra firme. Esto coincide con estudios que indican que los arrecifes más cercanos a la costa son los más vulnerables [22] ante perturbaciones por: tránsito marítimo, contaminación, sobrepesca y turismo [23]. La cobertura de coral en Mamey es similar al porcentaje promedio obtenido por la referencia [50] en Bocas del Toro (Caribe panameño). La cobertura de algas presentó un mayor porcentaje de cambio, ya que estas poseen un crecimiento muy acelerado a diferencia de los corales o las algas incrustantes [63], pero a su vez, por no estar tan fijas al sustrato su desprendimiento es más sencillo. En Playa Cacique se encontraron mayores porcentajes de algas que Mamey. Se sugiere que puede deberse a un mayor flujo de nutrientes proveniente de las actividades agrícolas de tierra firme [56], muy características de la zona de estudio. Es importante seguir con el monitoreo en el área para observar ciertas tendencias en los porcentajes para poder determinar si existe la posibilidad de una eutrofización en Playa Cacique.

El estado de deterioro de los corales en Playa Cacique puede deberse a que los primeros dos transectos quedaron frente a la playa, en donde se vierten los desechos de la comunidad [13]. Los últimos transectos en Cacique estuvieron frente a un pequeño litoral rocoso alejados de la comunidad, además estuvieron colonizados en su mayoría por coral fuego (Millepora) especies de gran importancia para los peces juveniles [63]. En Mamey el estado de los arrecifes puede estar afectado por la eliminación de las hierbas marinas en algunas áreas de la isla, lo que aumenta la turbidez en los arrecifes, hecho que aumenta la mortalidad de estos [64]

Cacique presentó 13 de las 14 especies encontradas en los muestreos. Mamey, en cambio, presentó solo siete, esto se puede deber a que en Mamey tres de los cuatro transectos (TM1, TM2 y TM3) estuvieron colonizados en su mayoría por los corales fuego, lo que disminuye la cantidad de especies presentes en los mismos. Además, la profundidad de cada transecto afecta la diversidad de corales presentes [9]. La familia más diversa fue la Faviidae, con un total de cinco especies, pero estos a su vez, solo están presentes en los transectos en donde la profundidad sobrepasaba el metro y medio. Es importante mencionar que la única especie que no estuvo presente en Cacique fue el A. palmata, especie de gran interés. La diversidad de corales encontrada (14 especies) es poca si la comparamos con la cantidad total de especies encontradas en el caribe panameño, siendo solo el $22 \%$ del total de 64 especies descritas por la referencia [50].

Los resultados de ictiofauna dieron un total de 21 especies de peces. Esta es una cantidad mínima de peces asociados a los arrecifes del caribe de Panamá [65-67]. Las Familias más diversas fueron: Labridae, Serranidae y Acanthuridae. En trabajos anteriores la familia Serranidae y Haemulidae eran 
dominantes en el Caribe [65-67], pero en las dos áreas de monitoreo eran escasas en cuanto a su abundancia. Las zonas estuvieron dominadas por $H$. pictus, presente en seis transectos. Además de ser la especie más abundante, esta especie se encontraba en aguas poco profundas de la costa.

También la especie $S$. diencaeus y $T$. bifasciatum fueron especies presentes en cuatro de ocho transectos, y se encontraban en zonas someras, sin importar el oleaje [68]. El oleaje y el clima no permitió definir la ictiofauna presente en las zonas de monitoreo. Por esta razón, los resultados no se asemejan a otros trabajos realizados en la costa caribeña de Panamá.

No existe una diferencia significativa en la abundancia y diversidad de especies de corales y peces entre las dos estaciones, esto posiblemente debido a que son lugares muy cercanos uno del otro que comparten características muy similares. Además, el número de salidas fue bajo como para poder hacer una caracterización de las áreas, por lo que es necesario seguir con los monitoreos.

\section{Conclusiones}

- Isla Mamey posee una mayor cobertura de coral vivo a comparación con Playa Cacique. Sin embargo, Playa Cacique posee un mejor estado en sus arrecifes, quizás por tener un ecosistema de manglar el cual mitiga la sedimentación en los arrecifes. Es posible que Isla Mamey tenga un peor estado, por tener un flujo de turistas mayor que Playa Cacique. Playa Cacique, por ser una comunidad con una población constante, genera muchos desechos que llegan al mar (desagües). Estos llegan a convertirse en nutrientes extras, lo que genera un aumento en las algas del sitio. En cambio, Mamey no posee descargas de agua provenientes de actividades antrópicas.

- Los corales del género Millepora son los más abundantes en las dos estaciones, esto debido a que son de crecimiento rápido y están adaptados para crecer en aguas someras. Observamos que entre más profundo era un arrecife, más diversidad de corales encontramos. La familia más diversa fue la Faviidae (corales cerebro) aunque solo se encontraron en los transectos más profundos. Solo se encontraron corales de la familia Agariciidae (coral lechuga) en los transectos de Playa Cacique.

- La ictiofauna asociada en los monitoreos fue escasa, debido a los fuertes oleajes. La especie más abundante fue el $H$. pictus, debido a que es la especie que está mejor adaptada para habitar aguas someras con fuertes oleajes. Se notó que la ictiofauna disminuía en los transectos en donde el coral fuego predominaba. Esto pudiera deberse a la naturaleza urticante del mismo. Isla Mamey presentó una mejor diversidad de peces que Playa Cacique, posiblemente por ser una isla que tiene interacciones con arrecifes de aguas profundas.

\section{AGRADECIMIENTO}

Agradecemos a la Universidad Marítima Internacional de Panamá (UMIP) por permitirnos realizar esta investigación, brindándonos los equipos y el transporte. Al profesor Casas y a la profesora Beatriz Medina por aconsejarnos en las giras y en la elaboración del trabajo. Al señor Adriano por brindarnos el servicio de transporte marítimo hacia Isla Mamey. También al Dr. Juan Posada por ayudarnos a identificación algunas especies de peces. A nuestra familia por permitirnos estudiar esta carrera y apoyarnos en todo momento.

\section{REFERENCIAS}

[1] K. Töpfer. "Conventions and Coral Reefs.” UNEP Coral Reef UnitWWF Coral Reefs Advocacy Initiative. Gland, Switzerland: UNEP Press. 2003.

[2] C.Wilkinson y D. Souter. "Status of Caribbean Coral Reefs after Bleaching and Hurricanes in 2005". Townsville, Australia: Global Coral Reef Monitoring Networt y Rainforest Research Center. 2008.

[3] Cortés, Jorge et. al. "Pérdida de zooxantelas y muerte de corales y otros organismos arrecifales en el Caribe y Pacífico de Costa Rica”. Revista de Biología Tropical. vol.32 (2), pp. 227-231, 1984.

[4] F. Moberg y C. Folke. "Ecological goods and services of coreal reef ecosystems." Ecol. Econ. 29 (2), pp. 215-233, 1999.

[5] P. Principe, P. Bradley, S. Yee, W. Fisher, E. Johnson, P. Allen, D. Campbell. "Quantifying coral reef ecosystem services." U.S. Environmental Protection Agency, Office of Research and Development, Research Triangle Park, NC. EPA/600/R-11/206. 2012.

[6] C. I. Elliff y I. R. Silva. "Coral reefs as the first line of defense: Shoreline protection in face of climate change." Marine Environmental Research. vol. 127, pp. 148-154, 2017.

[7] UNEP/IUCN. "Coral reefs of the world." Vol. 1: Atlantic and Eastern Pacifico IUCN, Gland Switzerland and Cambridge, U.K./UNEP, Nairobi, Kenya. 373 p. 1988.

[8] J.P.G. Spurgeon. "The ecollomic valuation of coral reefs." Mar. Pollut. Bull. vol. 11, pp. 529-536, 1992.

[9] H. M. Guzmán, I. Holst "Inventario biológico y estado actual de los arrecifes coralinos a ambos lados del Canal de Panamá.” Rev. Biol. Trop. vol. 42(3), pp. 493-514, 1994.

[10] P. Freon y O. A. Misund. "Dynamics of Pelagic Fish Distribution and Behaviour: Effects on Fisheries and Stock Assessment." Oxford, Inglaterra: Fishing New Books. 1999.

[11] P. Castro y M. Huber. Biología Marina, 2007, pp. 298-299.

[12] T. F Goreau, T. McClanahan, R. Hayes y A. Strong. "Conservation of coral reefs after the 1998 Global Bleaching." Event. Conserv. Biol., Vol. 14(1), pp. 5-15, 2000.

[13] T. F. Goreau "Mass expulsion of zooxanthellae from Jamaican reef cornmunities after Hurricane Flora." Science. Vol. 145, pp. 383-386, 1964.

[14] W. C. Jaap. "Observations on Zooxanthellae expulsion at Middle Sambo Reef, Florida Keys.” Bull. Mar. SeL. vol. 29, pp. 414-422, 1979.

[15] P. Garrett y H. Ducklow. "Coral diseases in Bermuda." Nature vol. 253, pp. 349-350, 1975.

[16] A. Antonius "Coral reef pathology: A review". Proc. 4th. Int. Coral Reef Sym. vol. 2, pp. 3-6, 1981. 
[17] A. Antonius "The "band" diseases in coral reefs." Proc. 4th. Int. Coral Reef Symp. vol. 2, pp. 7-14, 1981.

[18] T. F. Cooper, J. P. GilmourK. E. Fabricius. "Bioindicators of changes in water quality on coral reefs: review and recommendations for monitoring programmes." Coral Reefs, vol. 28(3), pp. 589-606, 2009.

[19] A. Dikou. "Ecological Processes and Contemporary Coral Reef Management." Diversity, vol. 2(5), pp. 717-737, 2010.

[20] K. E. Fabricius, G. De'ath, S. Noonan, S. Uthicke. "Ecological effects of ocean acidification and habitat complexity on reefassociated macroinvertebrate communities." Proc. R Soc. B, 281, 2013.

[21] Y. Cabrales, S. P. González "Evaluación de indicadores ecológicos en las comunidades de corales hermatípicos de la costa sur-central, provincia de cienfuegos, Cuba." Revista Investigaciones Marinas, vol. 1 (35), pp. 37-57, 2015.

[22] L. Burke, K. Reytar, M. Spalding \& A. Perry "Reefs at Risk." Washington, D. C., EE. UU.: World Resources Institute. 2011.

[23] G. Horta-Puga "Impactos ambientales." En J. W. Jr. Tunnell, E. A. Chávez \& K. Withers (Eds.), Arrecifes Coralinos del Sur del Golfo de México (pp. 183-205). México: Instituto Politécnico Nacional. 2010.

[24] H. Caballero, P. M. Alcolado, A. Semidey "Condición de los arrecifes de coral frente a costas con asentamientos humanos y aportes terrígenos: el caso del litoral habanero, Cuba." Rev. Mar. Cost, vol. 1. pp. 49-72, 2009.

[25] M. González-González, V. De la Cruz-Francisco, I. MoralesQuijano, R. E. Orduña-Medrano "Diversidad y cobertura de la comunidad bentónica del arrecife Enmedio, Sistema Arrecifal Lobos-Tuxpan, México.” Rev. Mar. Cost, vol. 8 (2), pp. 47-65, 2016.

[26] B. G. Hatcher. "Coral reef primary productivity: a beggar's banquet." Trends Ecol. Evol. vol. 3, pp. 106-111, 1988.

[27] B. G. Hatcher. "Coral reefs primary productivity: a hierarchy of partem and process." Trends Ecol. Evol. vol. 5, pp. 149-155, 1990.

[28] G. P. Jones. "Post recruitment process in the ecology of coral reef populations: a multifactorial perspective", pp. 294-328, 1991.

[29] J. P. Doherty. "Spatial and temporal patterns in recruitment", pp.2261-293, 1991.

[30] P. L. Munday, G. P. Jones. \& M. J. Caley. "Interspecific competition and coexistence in a guild of coraldwelling fishes". Ecology, vol 82, pp. 2177-2189, 2001.

[31] S. J. Holbrook \& R. J. Schmitt. "Competition for shelter spaces causes density dependent predation mortality in damselfishes". Ecology. vol.83, pp. 2855-2868, 2002.

[32] C. J. Fulton., D. R. Bellwood \& P. C. Wainwright. "Wave energy and swimming performance shape coral reef fish assemblages". Proceedings of the Royal Society. vol. 272, pp. 827-832, 2005.

[33] M. Srinivasan. "Depth distributions of coral reef fishes: the influence of microhabitat structure, settlement, and postsettlement processes". Oecologia. vol. 137, pp. 76-84, 2003.

[34] A. M. Friedlander, S. A. Sandin. DeMartini E. E., \& E. Sala. "Spatial patterns of the structure of reef fish assemblages at a pristine atoll in the central Pacific". Marine Ecology Progress Series. vol.410, pp. 219-231, 2010.

[35] I. Alemu, B. Jahson. "Fish assemblages on fringing reefs in the southern Caribbean: biodiversity, biomass and feeding types". Revista de Biología Tropical. pp. 169-181, 2014.
[36] T. P. Hughes. "Catastrophes, phase shifts and large-scale degradation of a Caribbean coral reef". Science, vol. 265, pp. 1547-1551, 1994.

[37] C. M. Roberts. "Effects of fishing on the ecosystem structure of coral reefs". Conservation Biology. vol.9, pp. 988-995, 1995

[38] A. L Green, D. R Bellwood \& H. Choat. "Monitoring functional groups of herbivorous reef fishes as indicators of coral reef resilience". A practical guide for coral reef managers in the Asia Pacific Region. Gland, Switzerland: IUCN, 2009.

[39] P. F. Sale. The science we need to develop for more effective management, 2002.

[40] R. S. Appeldoorn. C. W. Recksiek, R. L. Hill, F. E. Pagan. \& G. D Dennis. "Marine protected areas and reef fish movements: the role of habitat in controlling ontogenetic migration". Proceedings of the 8th International Coral Reef Symposium. vol. 2, pp. 19171922, 1997.

[41] K. N. Holland, J. D. Peterson, C. G. Lowe. \& B. M. Wetherbee. "Movements, distribution and growth rates of the white goatfish Mulloides flavolineatus in a fisheries conservation zone". The Bulletin of Marine Science. vol. 52, pp. 982-992, 1993.

[42] P.W. Glynn. "Observations on the ecology of the Caribbean and Pacific coasts of Panamá. Bull”. Biol. Soco Wash. vol. 2, pp. 1330, 1972.

[43] P.W. Glynn, RH. Stewalt \& J.E. McCosker. "Pacific coral reefs of Panamá: structure, distribution and predatorso Geol". Rundsch. vol. 61, pp. 483-519, 1972.

[44] J.W Porter. "Ecology and diversity of coral reefs on opposite sides of the isthmus of Panama. Bull". Biol. SOCo Wash. vol. 2, pp. 89-116, 1972.

[45] P.W. Glynn, "Coral communities and their modifications relative to past and prospective Central American seaway". Adv. Mar. Biol. vol.19, pp. 91-132, 1982.

[46] V.N. Zlatarski. "Porites colonensis, new species of stony coral (Anthozoa: Scleractnia) off the Caribbean coast of Panama. Proc". Biol. SOCo Wash. vol.103, pp.257-264, 1990.

[47] W.H. de Weerdt. \& P.W. Glynn. A new and presumably now extinct species of Millepora (Hydrozoa) in the Eastero Pacifico Zoologische Mededelingen vol. 65, pp. 267-276, 1991.

[48] N Knowlton, E. Weil, L.A. Weigh \& H.M. Guzmán. "Sibling specie ih Montastrea annularis , coral bleaching and the coral climate record”. Science vol.255, pp. 330-333, 1992.

[49] L. Holst. \& H.M. Guzmán. "Lista de corales hermatí (Anthozoa: Scleractinia; Hydrozoa: Milleporina) a ambos lados del istmo de Panamá”. Rev. Biol. Trop. vol.41, pp. 535-540, 1993.

[50] H. M. Guzmán y C. A. Guevara. "Arrecifes coralinos de Bocas del Toro, Panamá: 111. Distribución, estructura, diversidad y estado de conservación de los arrecifes de las islas Pastores, Cristóbal, Popa y Cayo Agua.” Rev. Biol. Trop., vol. 47(4), pp. 659-676, 1999

[51] Fondo Mundial para la Naturaleza The Importance of Coral to People. 2011.

[52] MarViva Compendio de Normas Pesqueras de la República de Panamá. 2011.

[53] Superficie, población y densidad de población en la República según provincia, comarca, distrito y corregimiento. Censos de 1990 a 2010. Instituto Nacional de Estadística y Censo (INEC) disponible https://www.contraloria.gob.pa/inec/SINAMP/pdf/colon.pdf Consultado el 27 de febrero de 2018. 
[54] Anónimo (2000) Portobelo riqueza natural y testimonio de glorias pasadas. Panamá América, 14 de junio del 2000, sección variedades.

[55] Diagnóstico de salud local del distrito de Portobelo, centro de salud señora Blasina Bernal Portobelo. Ministerio de salud región de Colon 2014. disponible en : http://www.minsa.gob.pa/sites/default/files/publicaciongeneral/a sis_local_portobelo_colon.pdf Consultado el 17 de marzo de 2018.

[56] Gaceta oficial (2017) Resolución No. DAPVS-0002-2017 de 28 de marzo de 2017. "Por el cual se apreuba el Plan de Uso Público del Parque Nacional Portobelo.

[57] J. M. Adames. Joya natural de Colón. Día a Día, 26 de septiembre del 2015, sección Tierra Adentro. 2015.

[58]Accuweather Cacique, Panamá disponible en: https://www.accuweather.com/es/pa/cacique/257775/februaryweather/257775?monyr=2/1/2018 Consultado el 17 de marzo de 2018.

[59] Climate-data.org, Portobelo, Panamá disponible en : https://es.climate-data.org/location/565445/ Consultado el 17 de marzo de 2018.

[60] L. R. Holdridge. "Zone Ecology”. Tropical Science Center. San José, Costa Rica. 1967.

[60] M.J. Shulman, \& D.R. Robertson. Changes in the coral reefs of San Blas, Caribbean Panama: 1983-1990. Coral Reefs 15: pp.231236,1996.

[61]Coralpedia (2018) Disponible en: http://coralpedia.bio.warwick.ac.uk/. Consultado el 13 de mayo del 2018

[62] D.R. Robertson, E. A. Peña, J. M. Posada y R. Claro "Peces Costeros del Gran Caribe: sistema de información en linea. Version 1.0 Instituto Smithsonian de Investigaciones Tropicales, Balboa, República de Panamá.

[63] I. Leal, P. Pereira. \& M. Araújo, "Coral reef fish association and behaviour on the fire coral Millepora spp. in north-east Brazil". Journal of the Marine Biological Association of the United Kingdom, vol. 93(6), pp. 1703-1711, 2013.
[64] C. S. Rogers. "The effect of shading on coral reef structure and function." Journal of Experimental Marine Biology and Ecology, vol. 41(3), pp. 269-288, 1979.

[64] R. T. J. L. Munro, "Aspects of the biology and ecology of Caribbean reef fishes: Serranidae (hinds and groupers)", Fish Biology, vol. 12, pp. 115-146, 1978.

[65] J. C. O. a. E. H. G. William B. Gladfelter, "Similarity and Diversity Among Coral Reef Fish Communities: A Comparison between Tropical Western Atlantic (Virgin Islands) and Tropical Central Pacific (Marshall Islands) Patch Reefs," Ecological Society of America, vol. 61, n 5, pp. 1156-1168, 1980.

[66] M. J. P.-C. Peter C. Phillips, "A comparative survey of reef fishes in Caribbean and Pacific Costa Rica," Biología tropical, vol. 32, 1984.

[67] J. a. S. B. Bohnsack, "A Stationary Visual Census Technique for Quantitatively Assessing Community Structure of Coral Reef Fishes," 1986.

[68] L. S. M. y. J. Garzón-Ferreira, "Estructura de comunidades de peces arrecifales en cuatro atolones del Archipiélago de San Andrés y Providencia (Caribe sur occidental)," Revista de biologia tropical, vol. 4, pp. 883-896, 2000. 